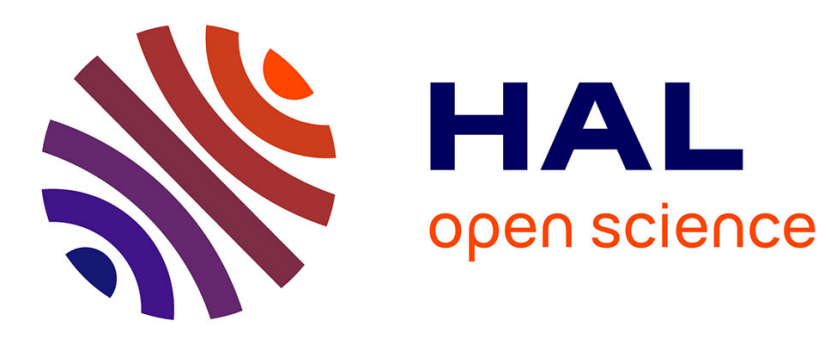

\title{
Spin Excitations of the Spin-Polarized Electron Gas in Semimagnetic Quantum Wells
}

B. Jusserand, F. Perez, D. R. Richards, G. Karczewski, T. Wojtowicz, C. Testelin, D. Wolverson, J. J. Davies

\section{- To cite this version:}

B. Jusserand, F. Perez, D. R. Richards, G. Karczewski, T. Wojtowicz, et al.. Spin Excitations of the Spin-Polarized Electron Gas in Semimagnetic Quantum Wells. Physical Review Letters, 2003, 91 (8), pp.086802. 10.1103/PhysRevLett.91.086802 . hal-00002783

\section{HAL Id: hal-00002783 https://hal.science/hal-00002783}

Submitted on 1 Jun 2016

HAL is a multi-disciplinary open access archive for the deposit and dissemination of scientific research documents, whether they are published or not. The documents may come from teaching and research institutions in France or abroad, or from public or private research centers.
L'archive ouverte pluridisciplinaire HAL, est destinée au dépôt et à la diffusion de documents scientifiques de niveau recherche, publiés ou non, émanant des établissements d'enseignement et de recherche français ou étrangers, des laboratoires publics ou privés. 


\title{
SPIN EXCITATIONS OF THE SPIN-POLARISED ELECTRON GAS IN SEMI-MAGNETIC QUANTUM WELLS
}

\author{
Bernard Jusserand $^{\mathrm{a}}$, F. Perez ${ }^{\mathrm{a}}$, David Richards ${ }^{\mathrm{b}}$, G. Karczewskic, T. Wojtowicz ${ }^{\mathrm{c}}$, \\ C.Testelin $^{\mathrm{d}}$, D.Wolverson ${ }^{\mathrm{e}}$, and J.J.Davies ${ }^{\mathrm{e}}$ \\ a- CNRS/LPN, Route de Nozay, 91460 Marcoussis, France, \\ b- Dept. of Physics, King's College London, Strand, London WC2R 2LS, UK \\ c-Inst. of Physics, Polish Academy of Sciences, Al.Lotnikow 32/46, Warszawa, Poland \\ d- GPS, Universités Paris 6 et 7, 2 Place Jussieu, 75251 Paris Cedex 05, France \\ e-Dept. of Physics, Univ. of Bath, UK
}

Collective and single-particle spin-flip excitations of a two-dimensional electron gas in a semimagnetic $\mathrm{Cd}_{1-\mathrm{x}} \mathrm{Mn}_{\mathrm{x}} \mathrm{Te}$ quantum well are observed by resonant Raman scattering. Application of a magnetic field splits the spin-subbands and a spin-polarisation is induced in the electron gas. Above $1 \mathrm{~T}$ the collective modes, which disperse with in-plane wavevector, dominate the spectra. The local spin-density approximation provides a good description of our results and enables us to confirm that the energy of the low wavevector collective mode is given by the bare Zeeman energy. 
High-mobility electron gases confined in semiconductor heterojunctions reveal new physics associated with electron-electron interactions in two dimensions. Spin-polarized electron gas systems are of particular interest, both because of the new information they are expected to provide about the nature of the exchange Coulomb interaction and correlations in an electron gas, and also because of their importance for spin-based electronics. Under an external magnetic field, a spin polarized two-dimensional electron gas (2DEG) can be achieved in which strong modifications of the exchange interactions are expected. However, most experimental investigations on 2DEG systems have been reported in the GaAs/GaAlAs system in which the $g$-factor, which governs the magnitude of the Zeeman spin-splitting, is very small. So in this system spin effects are only accessible with large external fields, at which point strong orbital quantization dominates the energy spectrum. Indeed, in Raman scattering measurements of the electronic excitations of spin polarized electron gases confined in GaAs/GaAlAs heterostructures [1], both magnetoplasmons and spin-flip transitions appear simultaneously.

Semimagnetic quantum wells based on $\mathrm{Cd}_{1-x} \mathrm{Mn}_{x} \mathrm{Te}$ materials allow a completely new approach to this problem. A giant Zeeman splitting is induced in these materials by exchange interactions between the conduction electrons and those localized on Mn ions [2]. Under the application of moderate magnetic fields spin effects dominate over orbital quantization and the reverse situation to that of GaAs becomes accessible, with large filling factors and significant spin polarization. Moreover, the effect of exchange-correlation interactions on the energies of the collective excitations are expected to be more significant in $\mathrm{Cd}_{1-x} \mathrm{Mn}_{x} \mathrm{Te}$ because of the smaller Bohr radius in this compound $(6 \mathrm{~nm}$ in CdTe instead of $10 \mathrm{~nm}$ in GaAs). The aim of this letter is to present a theoretical model corresponding to this new situation and to demonstrate that it provides an excellent description of spin-flip excitations under external magnetic field, which become accessible to Raman scattering measurements 
thanks to the recent availability of high quality semimagnetic modulation doped quantum wells. In particular, we will show that the main features predicted within this model coexistence of single particle and collective excitations, in-plane dispersion of spin-flip excitations and Larmor's theorem — are successfully demonstrated in our experiments.

Even though the electron mobility in semimagnetic quantum wells remains below that in GaAs systems, modulation doped quantum wells with a reasonable quality have been achieved in the past few years [3] and we have reported recently [4] the observation by electronic Raman scattering of the single-particle excitations (SPE) and collective charge excitations (plasmons) of $2 \mathrm{DEGs}$ in $\mathrm{Cd}_{1-x} \mathrm{Mn}_{x} \mathrm{Te}$ modulation doped quantum wells, as reported previously in GaAs quantum wells [5]. This novel result demonstrated that degenerate electron gases exist at low temperature in semimagnetic quantum wells, despite the relatively large disorder induced, in particular, by alloy fluctuations. We report in this letter the observation of both collective and single-particle spin flip excitations under applied magnetic fields $B$ smaller than $1 \mathrm{~T}$. The collective excitations emerge from the single particle excitations at low fields and display a significant dispersion as a function of the in-plane wavevector, providing unambiguous evidence that they are associated with mobile electrons in the quantum well. This sets these new results apart from previous reports on spin flip Raman scattering in undoped and modulation-doped quantum wells with semimagnetic barriers [6] in which localized electrons are involved. The measured variations with the field and the measured in-plane dispersions compare well with theoretical predictions based on the polarizability of a 2D electron gas including exchange-correlation corrections as a perturbation.

Raman scattering is a well-established method for the investigation of the elementary excitations of electron gases in semiconductors and their dispersion with the in-plane wavevector $q$. At a given $q$, the SPE line at $B=0$ extends from 0 up to maximum energy 
$\hbar \omega_{S P 0}$ which increases with increasing $q\left(\hbar \omega_{S P 0}=\hbar v_{F} q\right.$ where $v_{F}$ is the Fermi velocity of the 2D gas); this energy range is shown in Fig.1a. The Raman scattering line shape can then be described by the imaginary part of the 2D polarisability. Assuming non-interacting electrons, the variations of the non-interacting polarisability $P_{0}(\omega, q)$ with $B$ can be simply understood on the basis of the giant Zeeman splitting in the conduction band of the $\mathrm{Cd}_{1}$ ${ }_{x} \mathrm{Mn}_{x} \mathrm{Te}$ quantum well. Under magnetic field, the conduction band splits into two spin subbands with, in the absence of carriers, the splitting described by a modified Brillouin function [7]. The single SPE spectrum observed at 0T is thus expected to split at non vanishing field into four new structures associated with spin conserving ( $\uparrow$ and $\downarrow \downarrow$ ) and spin flip ( $\downarrow$ and $\downarrow \uparrow$ ) transitions, which were degenerate at 0T. Spin conserving transitions are expected to vary smoothly due to the increase in Fermi velocity $v_{F}^{\uparrow}$ of the majority spin subband, and decrease in $v_{F}^{\downarrow}$ of the minority spin subband. On the contrary, spin flip transitions are expected to display large variations with the field. In particular the SPE spectrum related to excitations from the majority to the minority spin subbands is a broad line centered at the Zeeman splitting $\Delta E_{Z}$ and extending between $\Delta E_{Z}-\hbar v_{F}^{\uparrow} q$ and $\Delta E_{Z}+\hbar v_{F}^{\uparrow} q$; this energy range is shown in Fig.1b,c,d for different values of the Zeeman splitting.

However, this behaviour is modified significantly when exchange-correlation interactions are taken into account. The interacting polarizability $P(\omega, q)$ associated with spin flip transitions can be deduced within a perturbative approach, similar to the Random Phase Approximation, applied to spin conserving transitions [8]:

$$
P(\omega, q)=\frac{P_{0}(\omega, q)}{1-V_{X C}(q) P_{0}(\omega, q)}
$$

where $V_{X C}$ is the exchange-correlation interaction. Within the local spin density approximation, $V_{X C}$ is independent of $q$ and may be obtained from the derivative, with respect to the spin-density $s=n \uparrow-n \downarrow$, of the exchange-correlation potential. This expression for the 
spin-flip polarizability has been used extensively to describe inter-subband collective excitations in GaAs quantum wells [8]. Here we use it in a different context: i) spin subbands are considered here, instead of 'orbital' subbands resulting from confinement along the growth direction; and ii) the exchange-correlation interactions have to be considered at finite spin polarization instead of $s=0$ in GaAs quantum wells. Moreover, we have the unique possibility of controlling the subband spacing with an external parameter: the magnetic field. We have used the Gunnarsson and Lundquist parametrization [9] of the exchange-correlation potentials $\mu_{X C}^{\uparrow}$ and $\mu_{X C}^{\downarrow}$ in a 3D spin-polarized electron gas. After integration over the local electron density profile $n_{3 D}(z)$ along the growth direction, the $2 \mathrm{D}$ interaction $V_{X C}$ is given by:

$$
V_{X C}=\frac{1}{2}\left(\frac{\partial \mu_{X C}^{\uparrow}}{\partial s}-\frac{\partial \mu_{X C}^{\downarrow}}{\partial s}\right)=-\frac{0.4073}{n}\left[\frac{1}{r_{s}}-0.036-\frac{1.36}{1+10 r_{s}}\right] R_{y}^{*} \frac{1+\left(0.297 \frac{s}{n}\right)^{2}}{\left[1-\left(0.297 \frac{s}{n}\right)^{2}\right\rceil^{2}}
$$

where $n=n \uparrow+n \downarrow$ is the 2D electron density and $\left(r_{s} a_{B}^{*}\right)^{3}=\frac{3 a}{4 \pi n} \cdot a_{B}^{*}, R_{y}^{*}$ and $a$ are the effective Bohr radius, the effective Rydberg energy and the average width of the electron distribution along $\mathrm{z}$, respectively.

Due to exchange-correlations, a new pole appears in the interacting polarizability $P(\omega, q)$ when the denominator vanishes. This is the collective spin flip (SF) excitation which, because $V_{X C}$ is negative, is expected to lie below the single particle energies by an "excitonic correction”. The variation of the imaginary part of $P(\omega, q)$, which represents the Raman scattering intensity, is shown in Fig. 2 as a function of the Zeeman splitting. In the absence of any Zeeman splitting the SPE extends down to vanishing energy (see Fig. 1a) and the SF excitation is heavily damped, with exchange-correlation only resulting in a modification of the line shape of spin-flip SPE. For finite Zeeman splittings, an energy window opens below the spin-flip (inter-spin-subband) SPE, as shown in Fig.1, and the collective SF excitation becomes a well-defined excitation with a long lifetime. The SPE becomes strongly screened 
and the Raman spectra are dominated by the collective mode. Moreover, the spin flip collective line displays a downward energy dispersion when the in-plane wavevector is increased. At some finite value of $q$ it enters the energy range for SPE and becomes strongly damped, at which point one then recovers a broad Raman response similar to the SPE line, but with a lineshape modified by exchange-correlation interactions. As illustrated in Figs. 1 and 2, this situation takes place at higher wavevectors when the Zeeman splitting is increased.

One must emphasize that exchange-correlation interactions have to be taken into account from the beginning in the description of the spin-flip excitations. They induce: i) a shift of the spin-down and spin-up levels due to exchange self-energies; and ii) the "excitonic correction" to the collective transition between these two levels, described above. The first contribution leads to an increase of the level splitting, compared with that in an undoped quantum well, giving rise to an effective Zeeman splitting. The existence of such a correction has already been deduced [2] from the observation of a significant reduction of the critical field required for full spin-polarization, with respect to the value predicted from the Brillouin function. As a consequence, the non-interacting polarizability in Equ.1 has to include the increase of the effective level splitting though an effective density-dependent Zeeman splitting $\Delta E_{Z}^{\prime}$. The second contribution then leads to the reduction of the collective SF energy with respect to the effective level splitting. According to Larmor's theorem, one should expect that the SF energy coincides with the bare Zeeman splitting $\Delta E_{Z}$ at $q=0$ [10].

To demonstrate the validity of the above model, we have studied a 10 nm-thick $\mathrm{Cd}_{1 \text { - }}$ ${ }_{x} \mathrm{Mn}_{x}$ Te quantum well with a nominal Mn concentration $x=1.8 \%$. The barriers are made of $\mathrm{Cd}_{1-x} \mathrm{Mg}_{x} \mathrm{Te}$ with $15 \%$ of $\mathrm{Mg}$ and modulation doping is achieved by introducing iodine within the top barrier only. The spacer thickness is $40 \mathrm{~nm}$. From our previous Raman scattering experiments [4], we have deduced that the electron density amounts to $2.4 \times 10^{11} \mathrm{~cm}^{-2}$ whereas magneto-transmission results [2] are well reproduced assuming $n=2.25 \times 10^{11} \mathrm{~cm}^{-2}$ and $x=$ 
1.6\%. Raman spectra have been measured from a sample immersed in superfluid Helium using a Ti-sapphire laser with a typical power density of $10 \mathrm{~W} / \mathrm{cm}^{2}$; this was found to be sufficiently low to avoid heating the $\mathrm{Mn}^{2+}$ system [11]. Strong resonance with the $\mathrm{E}_{1} \mathrm{H}_{2}$ absorption edge [5] has been achieved with a laser energy close to $1.65 \mathrm{eV}$. With a laser energy close to $1.65 \mathrm{eV}$ a strong resonance has been achieved with the $\mathrm{E}_{1} \underline{\mathrm{H}}_{2}$ absorption edge [5]; the Fermi edge does not move significantly over the magnetic field range employed. The sample was mounted in the bore of a small superconducting solenoid providing up to $4.5 \mathrm{~T}$. Complementary experiments have been performed up to $6 \mathrm{~T}$ in a split-coil configuration. We have not shifted the laser energy for different magnetic fields as the Fermi edge does not move significantly over the range of experimental parameters employed. To measure the dispersion of the excitations, we have performed measurements at three different angles of incidence on the sample surface. The light propagation has been kept parallel to the field and the normal to the sample surface has been turned with respect to this common axis. As spin related effects are sensitive to the total field, while Landau quantization remains negligible at very small fields, we do not expect any specific consequence of this unusual configuration required for our Raman scattering experiments. We present in this letter Raman spectra measured with crossed linear polarizations of the incident and scattered light, in which spin flip excitations are known to be Raman active.

We show in Fig.3 Raman spectra obtained at a few different applied magnetic field and for two different angle of incidence $\left(20^{\circ}, q=5.72 \mu \mathrm{m}^{-1}\right.$ and $\left.70^{\circ}, q=15.72 \mu \mathrm{m}^{-1}\right)$. At zero field, the spectra display a single line very close to the laser energy, assigned to the SPE. As shown in Fig.1a, the energy $\hbar \omega_{S F 0}$ of this line increases with increasing $q$, although its dispersion shows a significant departure from the expected linear dispersion $\hbar \omega_{S F 0}=\hbar v_{F} q$, previously attributed [4] to alloy disorder in the quantum well. Its observation clearly demonstrates the existence of a Fermi edge for mobile electrons. In the presence of a 
magnetic field, the SPE Raman peak shifts towards higher energies and becomes well separated from the laser line. As can be seen clearly for $q=15.72 \mu \mathrm{m}^{-1}$, the SPE line shape does not change significantly but the overall intensity decreases strongly while a narrow peak, labeled SF in Fig.3, emerges from the low energy side of the structure around 0.5T. For larger fields the narrow peak completely dominates the spectra and above $5 \mathrm{~T}$ its dispersion saturates around $7 \mathrm{meV}$, as shown in the insert of Fig.4. At $20^{\circ}$, the same field variation is observed, the transition between the low field SPE and the large field SF taking place around 0.2T. Another peak is observed at about $0.4 \mathrm{meV}$ for $20^{\circ}$; the energy of this peak changes little with magnetic field. It is likely to correspond to spin conserving processes (SPE or, possibly, the Landau damped acoustic plasmon of the partially spin-polarised system) but its observation should not be allowed with crossed polarization.

In Fig.4, we show the field dependence of the measured SPE and SF energies at the three different considered wavevectors. In the insert we show the SF energy variation at the smallest $q$ over a large range of fields. In agreement with the Larmor's theorem, this variation is well reproduced with a Brillouin function assuming $M n$ concentration $x=1.5 \%$, in excellent agreement with independent estimations, and a quite reasonable spin temperature, $\mathrm{T}=3.3 \mathrm{~K}$. Based on this fit, we have calculated, using the spin-flip interacting polarizability, the effective Zeeman energy $\Delta E_{Z}^{\prime}$ as well as the SF energies at different wavevectors $q$ as a function of magnetic field $B$. As can be seen in Fig.4, the measured SPE energies are very well reproduced, providing a strong validation of the simple exchange-correlation model considered in this work and further support for the validity of Larmor's theorem in this system. Extrapolating the SPE energy to larger fields, we predict a full polarization around 2.2 $\mathrm{T}$ (when the SPE energy matches the Fermi energy), in good agreement with independent estimates. On the other hand, the SF dispersion is overestimated. 
We also show in Figs. 1a-d the measured energies of both SPE and SF lines as a function of $q$, for $B=0.0,0.3,0.6$ and $0.8 \mathrm{~T}$ respectively, which we compare with the calculated dispersions of both the SPE and the SF energies. This figure further illustrates the good description of the Raman data provided by the spin-flip interacting polarizability. In particular, the critical wavevector at which the SF enters the SPE band and becomes overdamped compares well with the experimental observations (see Fig. 3) - at low fields the SF line is only present at low wavevector whereas at higher fields the critical wavevector is much higher and the SF line is observed for both low and high wavevectors. On the other hand, we observe again that the measured SF dispersions are systematically weaker than predicted by theory. There are a number of possible explanations for this discrepancy, such as a disorder induced increase of the SF damping. However, because of the simplicity of the exchange-correlation model, we have not attempted any further test of such explanations.

In conclusion, our Raman scattering investigation of 2D electron gases in semimagnetic quantum wells provided a detailed picture of the emergence of collective spin-flip excitations from the continuum of single particle transitions with increasing magnetic field. We deduce an exchange-correlation correction to the bare Zeeman splitting of typically 30\% for this electron density $\left(2.4 \times 10^{11} \mathrm{~cm}^{-2}\right)$ and we demonstrate that a local spin density approximation gives a reasonable account for this value. We have clearly demonstrated the collective nature of the narrow Raman line which dominates the Raman response above $0.5 \mathrm{~T}$ and have been able to describe well its in-plane dispersion. Though restricted to a paramagnetic quantum well, our study provides a powerful indication that collective spin-flip excitations should be the dominant excitation mechanism in both paramagnetic and ferromagnetic low-dimensional semiconductors.

We thank C.Gourdon (Groupe de Physique des Solides, Paris) for the loan of a superconducting magnet and to M.Potemski and J.Cibert for valuable discussions. 


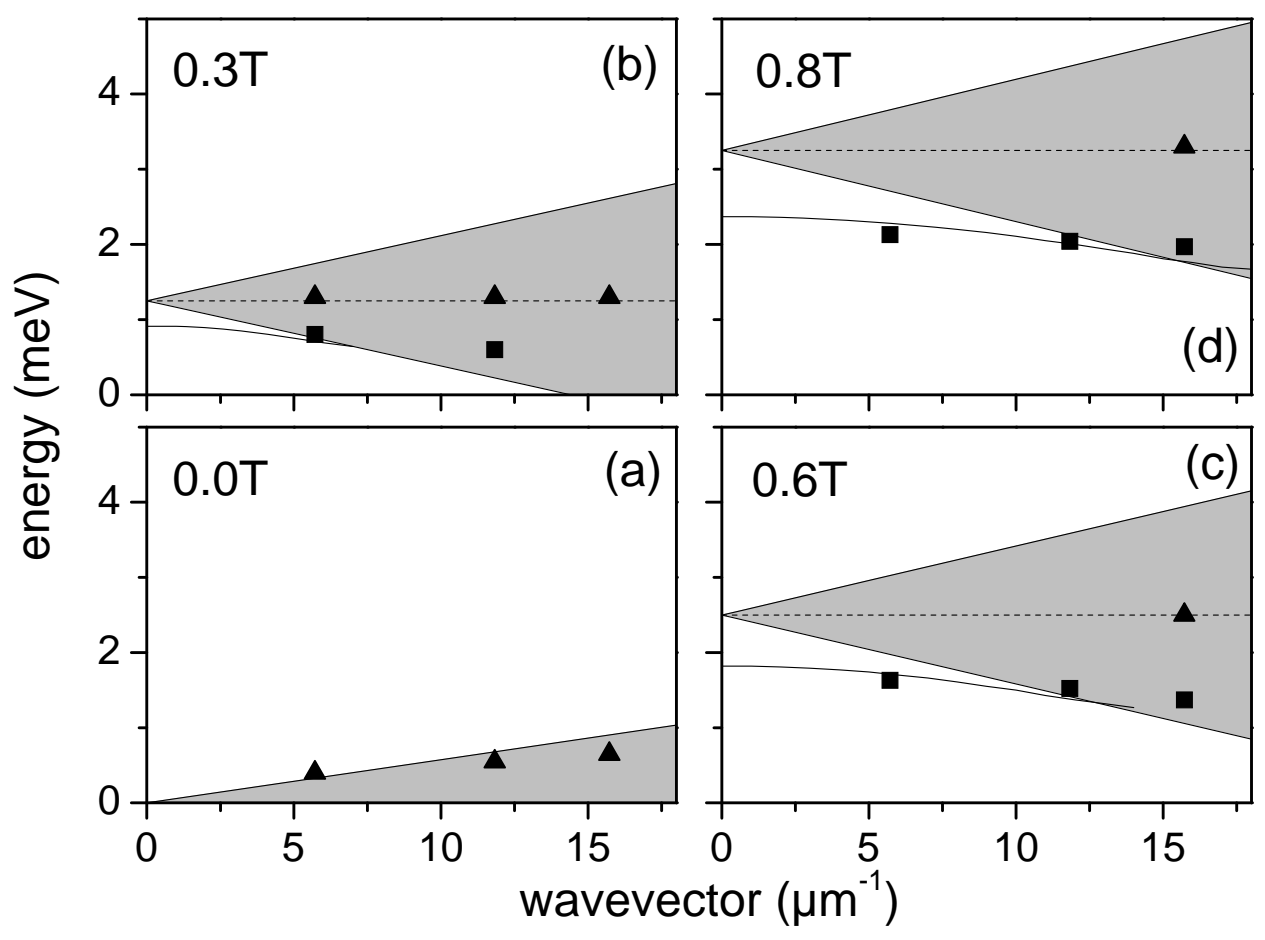

Fig.1 In plane dispersion of the SPE and SF excitations for four different values of the external field. For each field, experimental energies of the peak in the SPE band (triangles) and the SF line (squares) are compared, when available, to the mid-point (dashed line) in the calculated SPE energy range (shaded regions) and the SF energies in their wavevector range of existence(thick lines) 

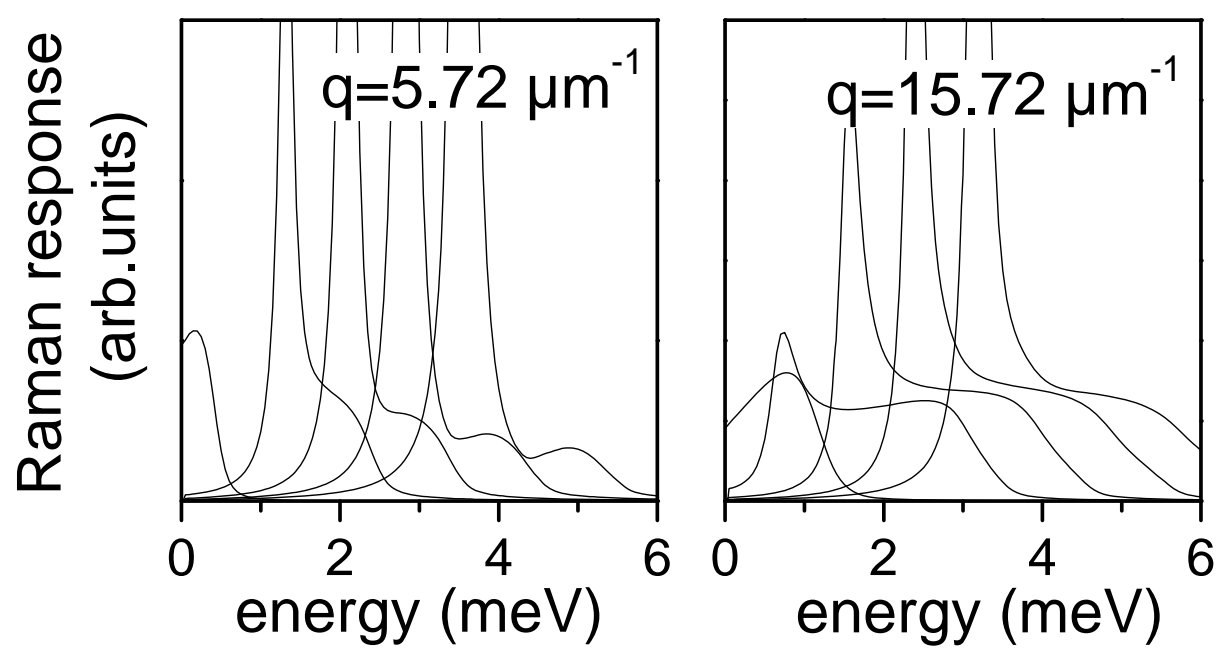

Fig.2 Raman response calculated from the interacting polarizability $P(\omega, q)$ for five different Zeeman energies $\left(\Delta E_{Z}=0,2,3,4,5 \mathrm{meV}\right)$ and for two different values of the transferred wavevector $q$. 


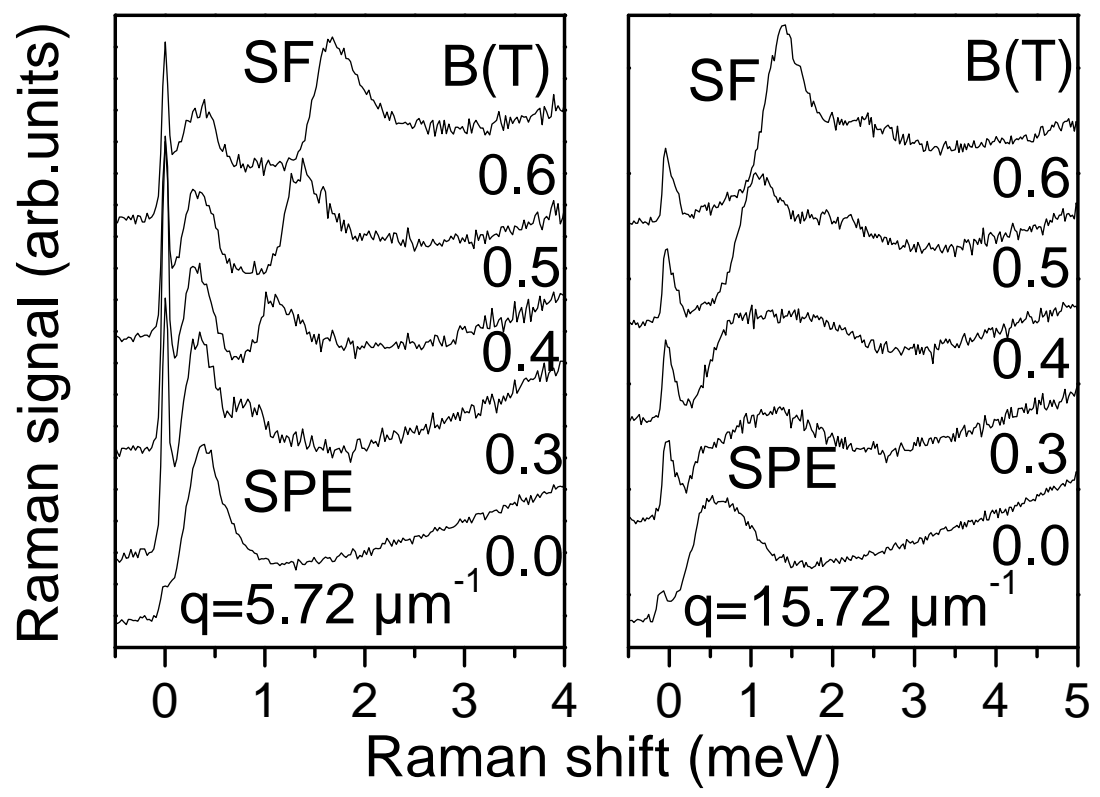

Fig.3 Raman spectra as a function of the external magnetic field and for two different values of the wavevector q transferred to the electron gas. The narrow line at vanishing Raman shift is the remnant of the laser line after the scattered light has passed through the subtractive premonochromator. 


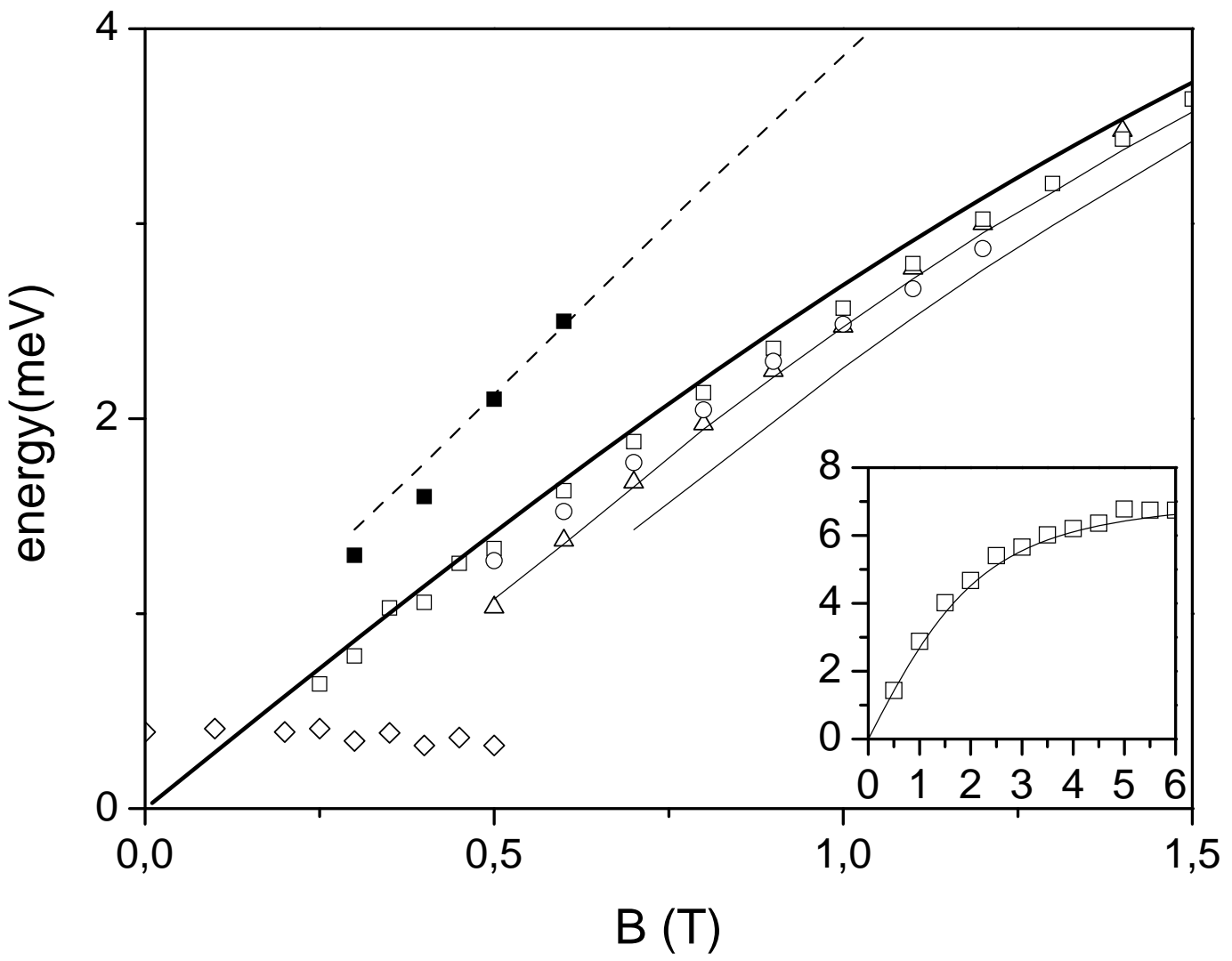

Fig.4 Variation with the applied magnetic field of the measured excitation energies: SPE ( full squares), SF excitation at $20^{\circ}$ (open squares), $45^{\circ}$ (open circles) and $70^{\circ}$ (open triangles). The diamonds indicate the energy of the low-energy line measured at $20^{\circ}$. The thick full line represents a Brillouin function fit of the SF line measured at $20^{\circ}$. The same fit is illustrated in the insert on a larger field range. The other lines show the field variations of the SPE energy (dashed line) and the SF energies at 45 and $70^{\circ}$ (thin narrow lines) as deduced from the model assuming the Brillouin dependence of the SF energy at $20^{\circ}$. 


\section{References:}

1. A. Pinczuk, et al., Phys. Rev. Lett. 68, 3623 (1992); H. D. M. Davies, et al., Phys. Rev. Lett. 78, 4095 (1997)

2. A. Lemaitre, et al., Phys. Rev. B 62, 5059 (2000) and references therein

3. G. Karczewski, et al., J. Cryst. Growth 184/185, 814 (1998)

4. B. Jusserand, et al., Phys. Rev. B 63, 161302(R), 2001

5. B. Jusserand, et al., Phys. Rev. Lett. 85, 5400 (2000) and references therein

6. M.P. Halsall, et al., Phys.Rev.B 50, 11755 (1994)

7. J. A.Gaj, R. Planel, and G. Fishman, Solid State Comm. 29, 435 (1979)

8. D. Gammon, et al., Phys. Rev. B 41, 12311 (1990)

9. O. Gunnarsson, and B. I. Lundqvist, Phys. Rev. B 13, 4274 (1976) and the erratum in Phys. Rev. B 15, 6006 (1977)

10. I.V. Kukushkin, et al., Phys. Rev. Lett. 85, 3688 (2000)

11. D.Keller, et al., Phys.Rev.B 65, 035313 (2002) 